\title{
SOME EXTENSIONS OF HILBERT'S INTEGRAL INEQUALITY
}

\author{
L. E. AZAR
}

Abstract. In this paper we introduce a new extension of Hilbert's integral inequality with a best constant factor involving the hypergeometric function. The equivalent form and some examples will be given.

Mathematics subject classification (2010): 26D15.

Keywords and phrases: Hardy-Hilbert's integral inequality, Holder's inequality, weight function, equivalent form.

\section{REFERENCES}

[1] G.H. Hardy, J.E. Littlewood, and G. Polya, Inequalities, Cambridge Univ. Press, London, 1952.

[2] Bicheng YANG, On Hilbert's Inequality with some parameters, Acta Math. Sinica (Chin.ser.), 49, 5 (2006), 1121-1126.

[3] Bicheng YAnG, On Best extensions of Hardy-Hilbert's inequality with two parameters, J. Ineq. in pure and Appl. Math., 6, 3 (2005), Art 81, 1-15.

[4] Mario Krnić, Gao Mingzhe, Josip Pecarić and Gao Xuemei, On the best constant in Hilbert, $s$ inequality, Math. Ineq. and Appl., 8, 2 (2005), 317-329.

[5] Mario Krnić, Hilbert Inequality and Gaussian Hypergeometric Functions, Journal of Math. Ineq., 3, 4 (2009), 645-656.

[6] M. Abramowitz, And I.A. Stegun, Handbook of Mathematical Functions With Formulas, Graphs, and Mathematical Tables, 9th printing, Dover, New York, 1972, pp. 807-808. 